\title{
Evaluation of Muscular Atrophy and Fatty Infiltration Using Time-zero Magnetic Resonance Imaging as Baseline Data, After Rotator Cuff Repair
}

\author{
Hyoung Bok Kim, Jae Chul Yoo ${ }^{1}$, Jeung Yeol Jeong ${ }^{2 \bowtie}$ \\ Department of Orthopedic Surgery, Bumin Hospital Seoul, ${ }^{1}$ Department of Orthopedic Surgery, Samsung Medical Center, Sungkyunkwan University College of \\ Medicine, Seoul, ²Department of Orthopedic Surgery, Hallym University Dongtan Sacred Heart Hospital, College of Medicine, Hallym University, Hwaseong, Korea
}

Background: This study evaluated postoperative changes in the supraspinatus from time-zero to 6 months, using magnetic resonance imaging (MRI). We hypothesized that restoration of the musculotendinous unit of the rotator cuff by tendon repair immediately improves the rotator cuff muscle status, and maintains it months after surgery.

Methods: Totally, 76 patients (29 men, 47 women) with rotator cuff tears involving the supraspinatus tendon who underwent arthroscopic rotator cuff repairs were examined. MRI evaluation showed complete repair with intact integrity of the torn tendon at both time-zero and at 6 months follow-up. All patients underwent standardized MRI at our institution preoperatively, at 1 or 2 days postoperative, and at 6 months after surgery. Supraspinatus muscular (SSP) atrophy (Thomazeau grade) and fatty infiltrations (Goutallier stage) were evaluated by MRI. The cross-sectional area of SSP in the fossa was also measured.

Results: As determined by MRI, the cross-sectional area of SSP significantly decreased $11.41 \%$ from time-zero (immediate repair) to 6 months post-surgery, whereas the Goutallier stage and Thomazeau grade showed no significant changes $(p<0.01)$. Furthermore, compared to the preoperative MRI, the postoperative MRI at 6 months showed a no statistically significant increase of $8.03 \%$ in the crosssectional area. In addition, morphological improvements were observed in patients with high grade Goutallier and Thomazeau at timezero, whereas morphology of patients with low grade factors were almost similar to before surgery.

Conclusions: Our results indicate that cross-sectional area of the initial repair appears to decrease after a few months postoperatively, possibly due to medial retraction or strained muscle.

(Clin Shoulder Elbow 2019;22(2):70-78)

Key Words: Muscular atrophy; Fatty infiltration; Rotator cuff repair; Time-zero magnetic resonance imaging

\section{Introduction}

Tendon tear of the rotator cuff alters the muscular structure. Over time, the musculotendinous units of the torn tendon retract and the muscle undergoes atrophy, fatty infiltration, and fibrosis, with loss of contractility and elasticity. ${ }^{1,2)}$ Muscular atrophy and fatty infiltration are recognized as important characteristics of rotator cuff tendon tear. They are associated with the severity and chronicity of the tendon tear, ${ }^{1)}$ resulting in decreased optimal repair, ${ }^{3)}$ inferior clinical outcome, and higher re-tear rate. ${ }^{4)}$ More recently, muscular atrophy and fatty infiltration are indicated as important prognostic factors in the anatomical and functional outcomes of rotator cuff repairs. ${ }^{1,3-6)}$

Regression or progression of muscular atrophy and fatty infiltration after cuff repair has gained momentum as a debatable issue. Some researchers suggest that muscle status can be improved after successful rotator cuff repair, ${ }^{5-8)}$ whereas others argue there is no improvement of muscle status despite com-

Received December 31, 2018. Revised February 3, 2019. Accepted February 6, 2019.

Correspondence to: Jeung Yeol Jeong

Department of Orthopedic Surgery, Hallym University Dongtan Sacred Heart Hospital, College of Medicine, Hallym University, 7 Keunjaebonggil, Hwaseong 18450, Korea

Tel: +82-31-8086-2410, Fax: +82-31-8086-2029, E-mail: inzaghy@naver.com, ORCID: https://orcid.org/0000-0001-8145-1302

IRB approval: Samsung Medical Center (No. SMC: 2016-02-019).

Financial support: None. Conflict of interests: None. 
plete and successful repair. ${ }^{4,9,10)}$ To address this issue in the clinical setting, past studies have compared preoperative computed tomography (CT) images or magnetic resonance imaging (MRI) with postoperative images at several months or years after repair.

However, previous results have an inherent limitation: the retraction of a tear can alter the comparability among different MRI scans. ${ }^{11)}$ Repairing a torn tendon acutely restores the musculotendinous unit of the rotator cuff. ${ }^{5-7,11)}$ This immediate change in muscle status after repair and subsequent healing process may preclude direct comparison of the muscle status between preoperative and postoperative MRIs months or years after surgery. Although recent literature shows that reversal could occur from 6 months to 2 years, the postoperative period from time-zero to 6 months has not been studied previously. ${ }^{12)}$ Furthermore, it remains to be determined if the initial repair can withstand the 6 months early rehabilitation period, or if there is a change in repair integrity.

This study was therefore undertaken to evaluate the postoperative changes in the supraspinatus muscle from time-zero to 6 months. We hypothesized that restoration of the musculotendinous unit of the rotator cuff by tendon repair would improve the rotator cuff muscle status immediately after surgery, and maintain it months after surgery.

\section{Methods}

\section{Patient Selection}

From July 2010 to October 2011, 76 patients who underwent arthroscopic rotator cuff repair for degenerative rotator cuff tears were enrolled in this study. Patients with partial- and fullthickness rotator cuff tears involving the supraspinatus tendon, such as isolated supraspinatus tears or supraspinatus, infraspinatus, and/or subscapularis tears, were included. All patients underwent three MRI assessments at Samsung Medical Center: preoperatively, 1 day or 2 days after surgery, and at 6 months (mean) after surgery. Complete repair was achieved in all the enrolled cases: type I complete repair with the tendon mobilized to the far lateral end of the greater tuberosity covering nearly the entire original footprint, and type II complete repair, with less optimal coverage of the entire medial-lateral footprint, indicating that the tendon is mobilized and attached to less than half of the medial side of the greater tuberosity. ${ }^{3)}$ Following cases were excluded from the final analysis: re-tear, isolated subscapularis tear, calcific tendinitis, missing Y-view image on T2 sagittal oblique MRIs, partial repairs (type III or IV repair ${ }^{3)}$ ), patients who underwent latissimus dorsi transfer, and patients lacking either time-zero and/or 6-month follow-up MRI. Also excluded were patients with infection, revision, or concomitant systemic disease such as rheumatoid arthritis and systemic lupus erythematosus. The final study cohort comprised of 49 patients. This study was approved by Samsung Medical Center Institutional Review
Board (No. SMC: 2016-02-019).

\section{Magnetic Resonance Imaging Examination}

The 49 patients included in the study were examined preoperatively with MRI or magnetic resonance arthrography (MRA): 12 patients underwent non-contrast MRI, and 37 patients underwent indirect MRA. Based on the MRI protocol, patients admitted a day before the surgery underwent indirect MRA whereas outpatients underwent non-contrast MRI during the study period at our hospital. Recent literature has reported that indirect MRA is likely to produce a similar outcome as non-contrast MRI when diagnosing for supraspinatus and infraspinatus muscle tears, except for partial articular supraspinatus tendon avulsion lesion. ${ }^{13)}$ Since our study evaluated only the supraspinatus, we expected that the 2 different MRI methods would not influence our results. All patients underwent non-contrast MRI for timezero and 6-month follow-up. Non-contrast MRI was performed with a 3.0-T magnetic resonance imager (Gyroscan Intera Achieva; Philips Medical Systems, Best, The Netherlands) with a dedicated receive-only shoulder coil. Patients were imaged supine, with the humerus in a neutral position and the thumb pointing upward. For MRA, $0.1 \mathrm{mmol} / \mathrm{kg}$ of gadobutrol (Gadovist; Bayer Healthcare, Berlin, Germany) was injected into an antecubital vein. Immediately after injecting the contrast media, patients were instructed to exercise their shoulders for 15 minutes, followed by MRI being performed similar to the non-contrast MRI. Conventional two-dimensional MRIs were obtained with fat-

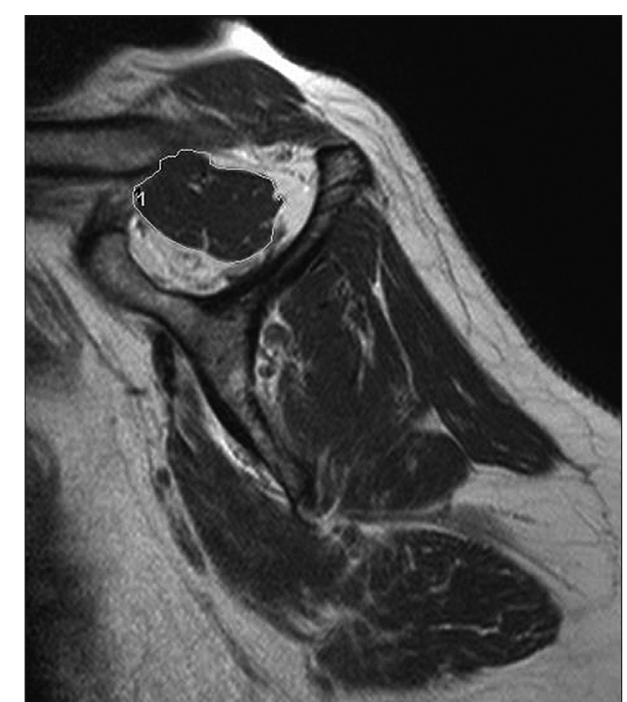

1 A $299.3 \mathrm{~mm}^{2}$ Average $487.7 \mathrm{~mm}^{2} \mathrm{SD} 187.4 \mathrm{~mm}$ Max 1,553.4 $\mathrm{mm}^{2}$ Min $69.0 \mathrm{~mm}^{2}$

Fig. 1. The area was measured using the Centricity-Radiology RA1000 workstation (GE Healthcare Integrated IT Solutions, Barrington, IL, USA) by use of a mouse-point cursor and automated computer calculation for distance and angle. All measurements were performed twice by 2 independent observers. SD: standard deviation, Max: maximum, Min: minimum. 
suppressed T1-weighted fast spin echo sequences in the axial and oblique coronal planes parallel to the long axis of the supraspinatus tendon, and oblique sagittal plane perpendicular to the long axis of the supraspinatus tendon (3-mm thick; 1-mm gap between slices; field of view, $15 \mathrm{~cm}$; matrix size of 224 pixels [224×224]; echo train length, 16). All measurements were performed on a picture archiving and communication system (PACS) monitor (GE Healthcare Integrated IT Solutions, Barrington, IL, USA) using a mouse-point cursor and automated computer calculation for distance and angle (Fig. 1).

\section{Magnetic Resonance Imaging Evaluation}

The results were evaluated by 2 independent orthopedic surgeons, blinded to each other to increase interobserver reliability. The Goutallier stage, Thomazeau grade, and occupation ratio of the supraspinatus were individually measured using the same image cuts. For intraobserver reliability, measurements were done at a 2-week interval period. The complete footprint coverage of the supraspinatus tendon was first assessed on a T2weighted coronal plane at time-zero MRI in all patients. This was followed by assessing the tendon integrity on a T2-weighted coronal plane in the 6 months postoperative MRI using the Sugaya classification. ${ }^{14)}$

\section{1) Fatty infiltration measured using the Goutallier stage}

Goutallier stages are classified as: stage 0, no fat; stage 1, some fatty streaks; stage 2 , less fat than muscle; stage 3 , equal amounts of fat and muscle; stage 4 , more fat than muscle. ${ }^{1,15)}$ For statistical analysis, each stage was given a score. Although originally described for $\mathrm{CT}$, previous studies have appropriately

Table 1. Intraobserver and Interobserver Reliability of Measures Used in the Study

\begin{tabular}{|c|c|c|c|}
\hline \multirow{2}{*}{ Variable } & \multirow{2}{*}{ Interobserver } & \multicolumn{2}{|c|}{ Intraobserver } \\
\hline & & Rater1 & Rater2 \\
\hline \multicolumn{4}{|l|}{ Preoperative MRI } \\
\hline Goutallier stage & 0.97 & 0.96 & 0.94 \\
\hline Thomazeau grade & 0.98 & 0.98 & 0.96 \\
\hline Cross-sectional area & 1.00 & 1.00 & 1.00 \\
\hline \multicolumn{4}{|l|}{ Time-zero MRI } \\
\hline Goutallier stage & 0.96 & 0.95 & 0.90 \\
\hline Thomazeau grade & 0.96 & 0.97 & 0.97 \\
\hline Cross-sectional area & 1.00 & 1.00 & 1.00 \\
\hline \multicolumn{4}{|l|}{6 months follow-up MRI } \\
\hline Goutallier stage & 0.96 & 0.96 & 0.89 \\
\hline Thomazeau grade & 1.00 & 0.92 & 0.92 \\
\hline Cross-sectional area & 1.00 & 1.00 & 1.00 \\
\hline
\end{tabular}

Interobserver and intraobserver reliability was excellent for all values. MRI: magnetic resonance imaging. applied this classification system to MRI. ${ }^{10)}$

2) Muscular atrophy measured using the Thomazeau grade

The Thomazeau grade is based on the occupation ratio of the supraspinatus with the supraspinatus fossa: grade 1, 0.6-1.0 (normal/slight); grade 2, 0.4-0.6 (moderate); grade 3, <0.4 (severe). ${ }^{8)}$ Atrophy of the supraspinatus was graded as 1, 2, and 3. For statistical analysis, normal and slight were given a score of 1 ; moderate, 2; and severe, 3.

\section{3) Muscle atrophy measured using cross-sectional area}

The cross-sectional area of the supraspinatus muscle in the supraspinatus fossa was measured using the freehand 'polygonal region of interest' tool on the PACS workstation. The T2weighted, sagittal oblique plane images of the point where the coracoids and the scapular spine meet the scapular body were used for all evaluations.

Table 2. Patient Demographics

\begin{tabular}{lc}
\hline \multicolumn{1}{c}{ Variable } & Value \\
\hline Age at surgery (yr) & $60.0 \pm 7.12(40-71)$ \\
\hline Gender (man/woman) & $14 / 35$ \\
\hline Duration of symptom (mo) & $23.61 \pm 23.85(1.81-121.94)$ \\
\hline Tear size & \\
\hline Partial & 10 \\
\hline Small & 10 \\
\hline Medium & 23 \\
\hline Large & 6 \\
\hline Dominant arm (Rt/Lt) & $47 / 2$ \\
\hline Involved side (Rt/Lt) & $35 / 14$ \\
\hline Dominant shoulder involved & $35(72.92)$ \\
\hline Repair technique (single row:double row) & $7: 42$ \\
\hline $\begin{array}{l}\text { Repair configuration intraoperatively } \\
\text { (Type I:Type II }{ }^{\dagger} \text { ) }\end{array}$ & $44: 5$ \\
\hline $\begin{array}{l}\text { Repair integrity at final follow-up } \\
\text { (Type I:Type II:Type III) }\end{array}$ & $4.98 \pm 0.43(1-3)$ \\
\hline $\begin{array}{l}\text { Mean duration of MRI follow-up (POD) } \\
\text { Time-zero MRI (d) }\end{array}$ & $28: 18: 3$ \\
\hline Follow-up MRI (mo) & $1.92(3.77-6.61)$ \\
\hline Total enrollment (2010.7-2011.10.) & 49 \\
\hline
\end{tabular}

Values are presented as mean \pm standard deviation (range), number only, number (\%), or ratio.

Rt: right, Lt: left, MRI: magnetic resonance imaging, POD: postoperative date. ${ }^{*}$ The tear size was classified according to the tear diameter, by applying the classification system of Goutallier et al. ${ }^{17)}$ as follows: small $(<1 \mathrm{~cm})$, medium $(1-3 \mathrm{~cm})$, large $(3-5 \mathrm{~cm})$, or massive $(>5 \mathrm{~cm})$.

${ }^{\dagger}$ The type of repair was classified according to the Yoo classification ${ }^{3)}$ : Type I: complete repair with the tendon mobilized to the far lateral end of the greater tuberosity covering nearly all of the original footprint; Type II: complete repair with the tendon mobilized to the medial one half or less of the greater tuberosity.

${ }^{*}$ Integrity in accordance with Sugaya classification. ${ }^{14)}$ 


\section{Clinical Evaluation}

All patients were clinically evaluated via the pain visual analogue scale (PVAS), function visual analogue scale (FVAS), American Shoulder and Elbow Surgeons (ASES) score, ${ }^{12)}$ and Constant score. ${ }^{16)}$ Clinical evaluation was performed a day before surgery, approximately 6 months after surgery, approximately 1 year after surgery, and at final follow-up (mean, 4 years). Those who were unable to visit the out-patient clinic had a telephone survey at the final follow-up. Clinical scoring was performed by a physiotherapist blinded to the study design or the clinical results.

\section{Operative Technique}

All surgeries were performed by a single senior surgeon (JCY). Patients were operated in a semi-lateral decubitus position and were administered regional anesthesia. Torn tendons were systematically identified, recorded, mobilized, and reattached to the greater tuberosity using suture anchors with a simple stitch, in a single-row fashion in 7 shoulders and double-row fashion with a suture bridge in 42 shoulders. Complete coverage of the rotator cuff footprint was achieved in all patients. Type I repair was performed in 44 shoulders and type II in 5 shoulders. All patients underwent a routine rehabilitation protocol, with immobilization for 4 weeks followed by active-assisted passive range of motion (ROM) exercises. Patients were subjected to stretching and active strengthening exercise after achieving full ROM.

\section{Statistical Analysis}

Continuous variables, including preoperative and postoperative clinical scores (PVAS, FVAS, ASES, and Constant score), were compared by analysis of variance (ANOVA). Changes in fatty infiltration, muscle atrophy, and cross-sectional area of the supraspinatus muscle between serial MRI studies were evaluated by repeated ANOVA using the SAS ver. 9.3 (SAS Institute, Cary, NC, USA). Interclass correlation coefficients between the 2 independent observers were analyzed by applying IBM SPSS Statistics ver. 21.0 (IBM Corp., Armonk, NY, USA). p-values were corrected by Bonferroni method in case of multiple testing, and considered significant less than 0.05 .

\section{Results}

Interobserver and intraobserver reliability were excellent for
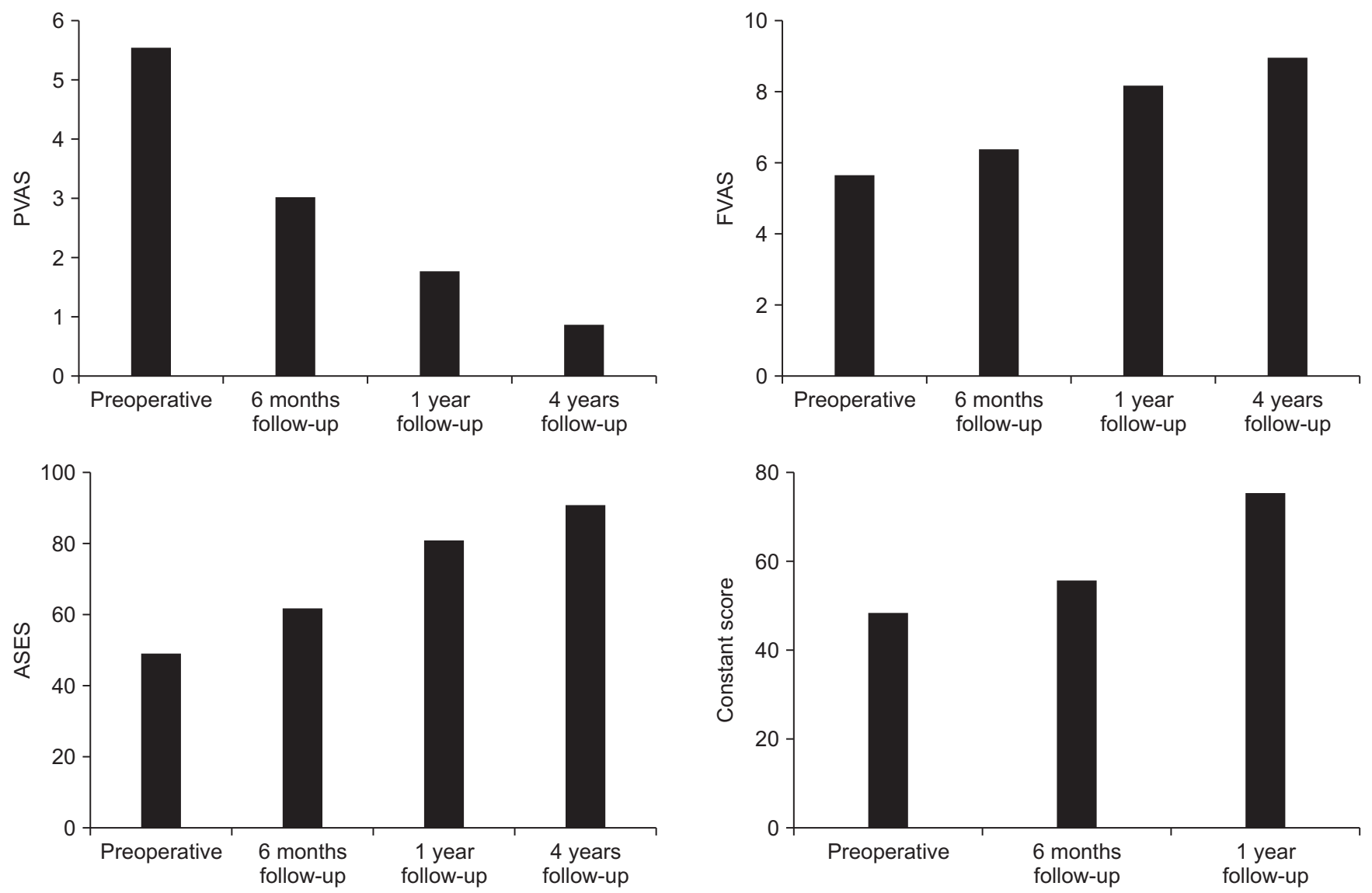

Fig. 2. The pain visual analogue scale (PVAS), function visual analogue scale (FVAS), and American Shoulder and Elbow Surgeons (ASES) were significantly improved from preoperative to postoperative 6 months $(p<0.001)$, from 6 months to 1 year $(p<0.001)$, and from preoperative to the final follow-up evaluations $(p<0.001)$. Constant scores were significantly improved from preoperative to postoperative 6 months $(p<0.001)$, and from 6 months to 1 year $(p<0.001)$. 
all factors evaluated (Table 1). In all patients, complete footprint coverage of the supraspinatus tendon was confirmed on T2weighted, coronal-plane MRI at time-zero, suggesting that the musculotendinous unit of the supraspinatus was successfully restored. Furthermore, Sugaya classification ${ }^{14)}$ confirmed the integrity of the repaired tendons on T2-weighted, coronal-plane MRI at postoperative 6 months. The baseline characteristics of the patients in our study are presented in Table 2.,14,17)

\section{Clinical Outcome}

Significant improvements were observed in the PVAS, FVAS, ASES, and Constant scores from preoperative to postoperative 6 months, from 6 months to 1 year, and from preoperative to the final follow-up evaluations (Fig. 2).

\section{Magnetic Resonance Imaging Evaluations}

1) Fatty infiltration measured using the Goutallier stage

From preoperative to time-zero, a significant improve- ment was observed in the Goutallier stage of the supraspinatus (1.58-1.39, respectively; $p=0.0034)$. However, no significant change was observed in the preoperative to 6-month follow-up MRI (1.58-1.30, respectively; $p=0.06)$ or in the time-zero to 6-month follow-up MRI (1.39-1.30, $p=0.93)$ (Fig. 3A). Notably, Goutallier stages 3 and 4 were significantly decreased after immediate rotator cuff repair $(p<0.01)$. Furthermore, we observed an increase in the relative distribution of stage $2(p=0.65)$, with no significant difference being observed for stage 0 and 1 $(p=0.85)$ (Fig. 3B).

\section{2) Muscle atrophy measured using the Thomazeau grade}

After immediate repair, the mean atrophy grade significantly improved from 1.90 to 1.67 ( $p=0.0015)$, but no significant differences were noted between preoperative and 6-month followup, or between time-zero and 6-month follow-up ( $p=0.60$ and $p=0.67$, respectively) (Fig. 3C). Immediately after rotator cuff repair, the number of patients with Thomazeau grade 3 muscle atrophy decreased significantly $(p<0.01)$, whereas the number of

B
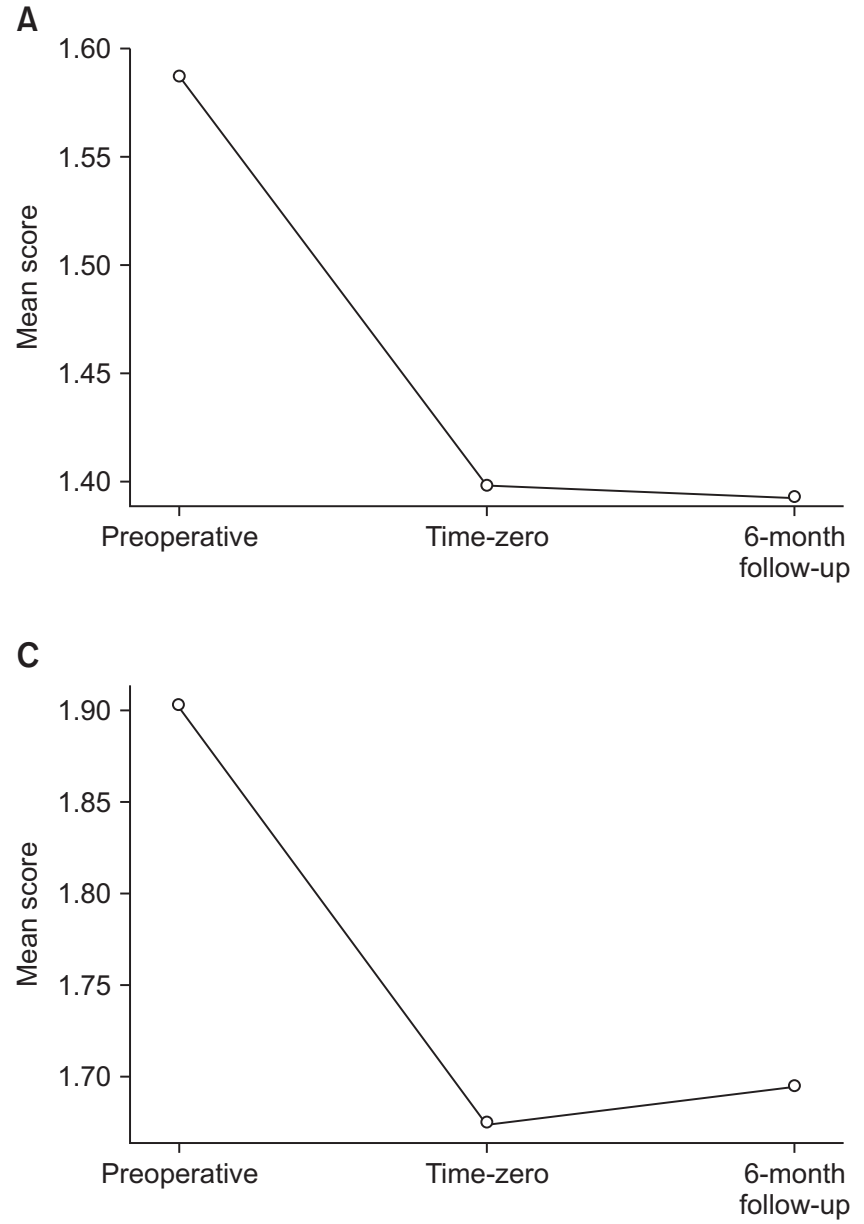

D

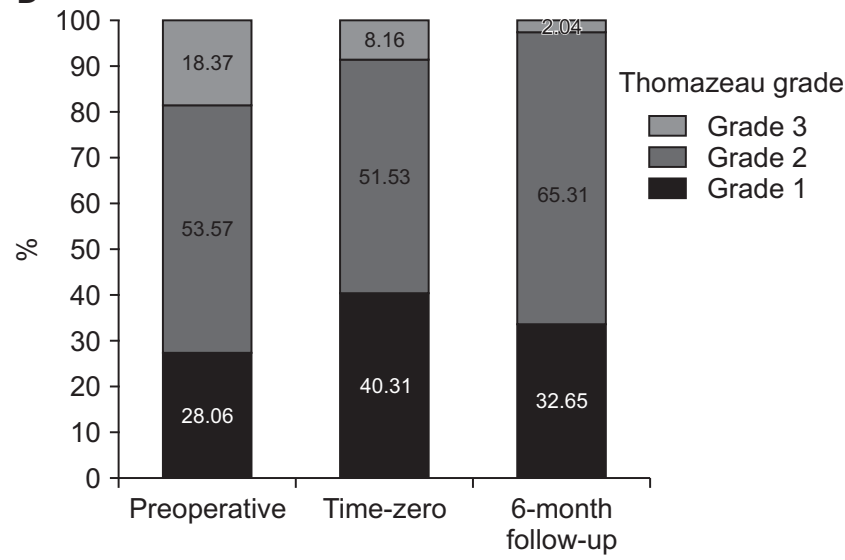

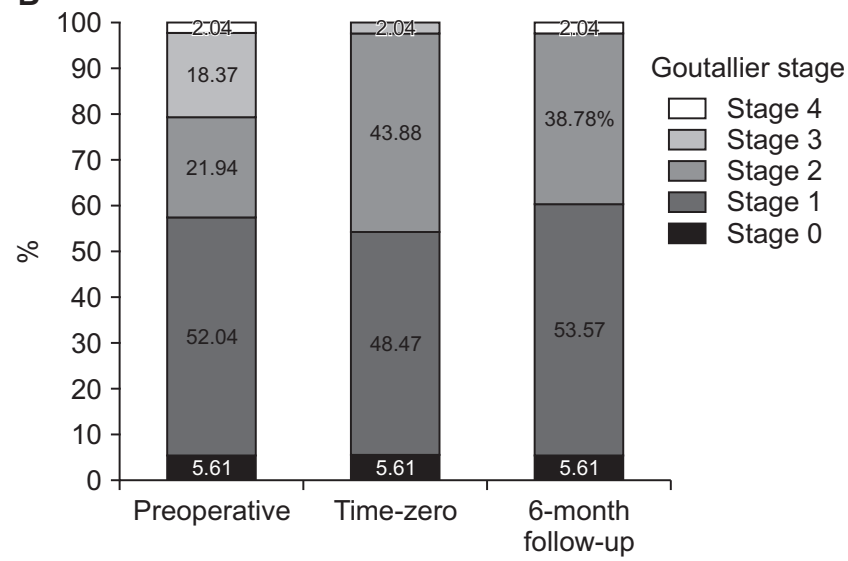

Fig. 3. (A) Goutallier stages were significantly improved from preoperative magnetic resonance imaging (MRI) to time-zero MRI ( $p=0.0034)$. However, no significant changes were observed between preoperative to 6-month follow-up MRI ( $p=0.06$ ), and time-zero to 6-month follow-up MRI ( $p=0.93$ ). (B) Goutallier stage 3 and 4 were significantly decreased from preoperative MRI to time-zero MRI $(p<0.01)$. (C) Thomazeau grade were significantly improved from preoperative MRI to time-zero MRI ( $p=0.0015)$, although there were no significant differences between preoperative and follow-up MRI, and time-zero and follow-up MRI $(p=0.60, p=0.67)$. (D) Thomazeau grades 3 were significantly decreased from preoperative MRI to time-zero MRI $(p<0.01)$. 
patients with Thomazeau grades 1 and 2 remained unchanged (Fig. 3D).

\section{3) Muscle atrophy measured using cross-sectional area}

The mean cross-sectional area of the supraspinatus significantly improved from $373.76 \mathrm{~mm}^{2}$ preoperatively to 446.12 $\mathrm{mm}^{2}$ at time-zero $(p<0.0001)$ (Fig. 4A). Immediately after surgery, the mean cross-sectional area increased to $21.72 \%$. MRI revealed significantly reduced cross-sectional area from timezero to 6 months follow-up ( 446.12 to $388.48 \mathrm{~mm}^{2}$, respectively; $11.41 \%$ reduction; $p<0.0001)$. Comparison of preoperative MRI with 6-month MRI showed an $8.03 \%$ increase in the crosssectional area, which was not statistically significant $(p=0.14)$ (Fig. 4B-D).

\section{Discussion}

This study was undertaken to evaluate changes in the morphology of fatty infiltration and muscle atrophy of rotator cuff muscles after arthroscopic rotator cuff repair by applying MRI.
Our MRI results revealed $11.41 \%$ decrease in the cross-sectional area of the supraspinatus muscle from time-zero (immediate repair) to postoperative 6 months, but no significant changes were observed in the Goutallier stage and Thomazeau grades. Compared with preoperative MRI, the postoperative MRI at 6 months (mean) shows only an $8.03 \%$ increase in the crosssectional area, which is statistically not significant. In addition, patients with high grade Goutallier and Thomazeau showed significant morphologic improvements at time-zero, whereas patients with low grade were almost similar to the pre-operative morphology.

Rotator cuff tear repair consistently results in decreased pain and increased shoulder function. However, the effects of repair on atrophy and fatty infiltration are still controversial. ${ }^{1,5,8,9,15)}$ Several studies using MRI have documented muscle atrophy reversal. Thomazeau et al. ${ }^{8}{ }^{8}$ observed that supraspinatus muscle atrophy improved in 18 of 22 rotator cuffs at 21 months after surgery, using a scoring system based on MRI signal intensities. ${ }^{18,19)}$ Gerber et al. ${ }^{7}$ showed an insignificant increase in the
A

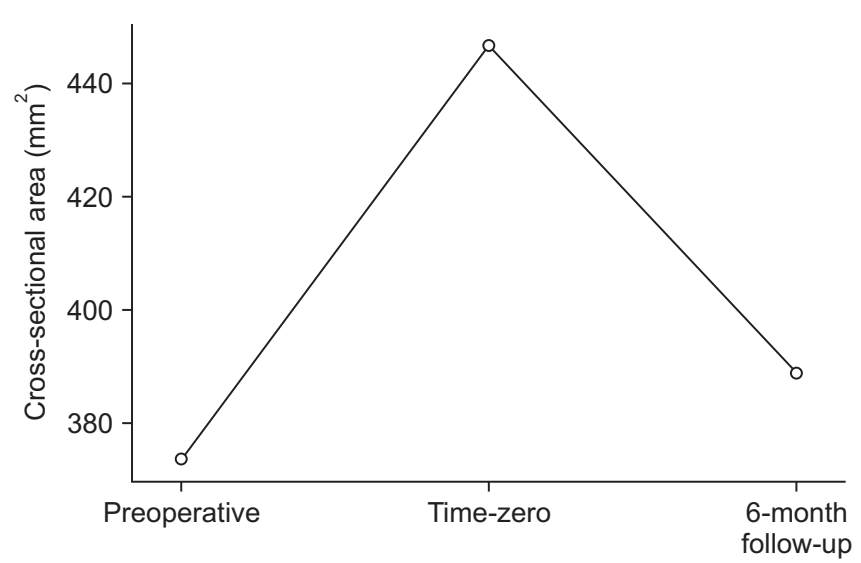

C

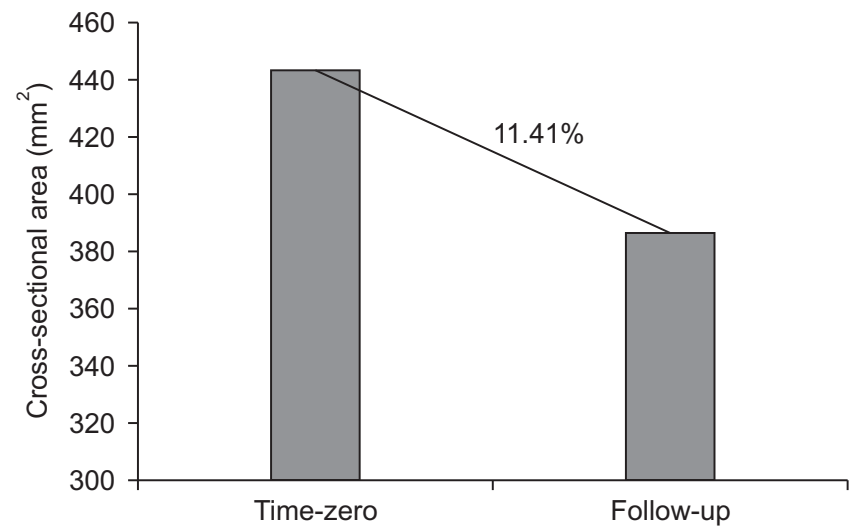

B

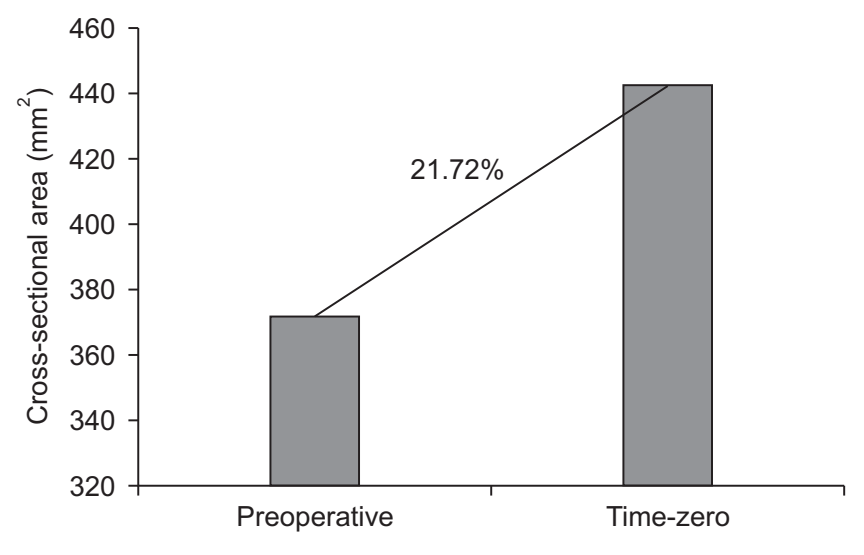

D

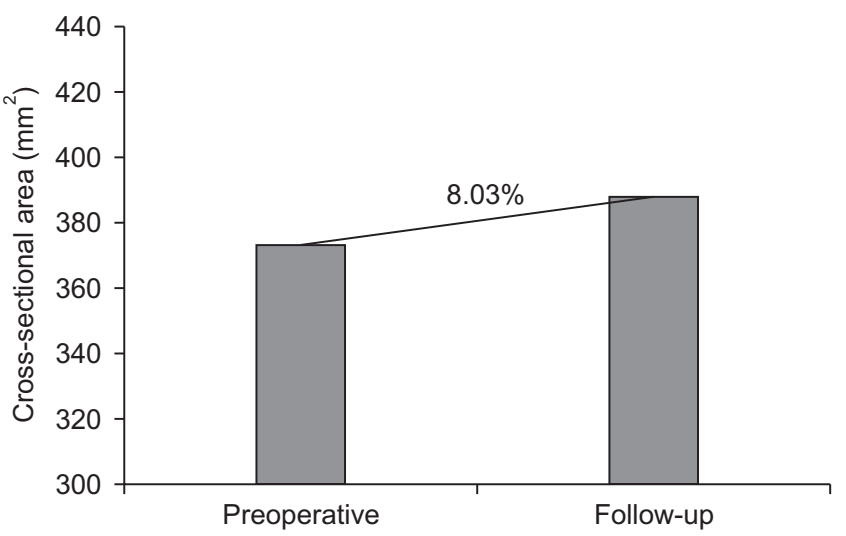

Fig. 4. (A) Cross-sectional area of the supraspinatus significantly improved from $373.76 \mathrm{~mm}^{2}$ preoperatively to $444.12 \mathrm{~mm}^{2}$ time-zero magnetic resonance imaging (MRI) $(p<0.0001)$. Cross sectional area of supraspinatus changes were: (B) increased $21.72 \%$ from preoperative MRI to time-zero MRI ( $p<0.0001)$, (C) decreased 11.41\% from time-zero MRI to follow-up MRI ( $p<0.0001)$, and (D) increased $8.03 \%$ from preoperative to follow-up MRI, although difference was statistically not significant $(p=0.14)$. 
cross-sectional area of the successfully repaired supraspinatus at 2 years, and concluded that atrophy may be reversed with successful repair. Goutallier et al. ${ }^{20)}$ found that at 18 months after successful repair, supraspinatus fatty infiltration was decreased in 6 of the 14 patients evaluated. Results of the current study suggest that even with grade 3 or 4 fatty infiltration, repair could result in improving the amount of atrophy and fat in the muscle. In an experimental study with rabbit models, Rubino et al. ${ }^{5)}$ reported that repair prevents further progression and atrophy of the rotator cuff.

Contrarily, Gladstone et al. ${ }^{9)}$ reported that at a minimum of 1 year, successful repair resulted in no morphologic improvement or reversal of muscle atrophy and fatty infiltration. Studies by Matsumoto et al. ${ }^{18)}$ and Shimizu et al. ${ }^{19)}$ showed no reversal of fatty infiltration at 6 and 12 months, respectively, after rotator cuff repair. However, these findings of atrophy reversal have an inherent limitation, as they were based on the comparison between preoperative and postoperative findings several months after repair, and the follow-up period was relatively short. Furthermore, these studies did not have baseline measurement after immediate repair and were instead compared with the preoperative muscle status.

Jo and Shin ${ }^{11)}$ investigated the changes in fatty infiltration and muscle atrophy by comparing preoperative MRI with time-zero MRI in 101 patients who underwent arthroscopic rotator cuff repair. They reported immediate improvement of fatty infiltration and rotator cuff atrophy after successful surgery. This study suggested that time-zero MRI provides the baseline data, which are clinically important in comparing changes in the muscle status. This finding coincides with our study results. However, their study did not include a longer follow-up period, especially the subsequent changes in fatty infiltration and muscle atrophy. ${ }^{11)}$

In our study, we observed changes in the muscle status using time-zero MRI as baseline data. Before commencing our study, we hypothesized that rotator cuff repair would improve the muscle status and maintain it months after surgery. However, MRIs from time-zero to 6-month follow-up reveal a decrease in the cross-sectional area, whereas the Goutallier stage and Thomazeau grade remain unchanged. McCarron et al. ${ }^{21)}$ undertook CT assessment of tendon retraction in 14 patients who underwent arthroscopic rotator cuff repair using Tantalum markers placed within the repaired tendons. This study showed that at postoperative 6 weeks, the repaired tendon significantly retracted medially without tear. They suggested that the medial retraction of the repaired tendon might be a natural phenomenon in patients who underwent rotator cuff repair. Baring et al. ${ }^{22)}$ reported that retraction of repaired tendon occurs 4 weeks after rotator cuff repair. This phenomenon in our study might explain why the cross-sectional area decreases at 6 months, compared to the MRI findings at time-zero (Fig. 5). Another possible explanation for reduced cross-sectional area might be that postoperative immobilization and non-weight-loading exercises cause further atrophy of the repaired tendon. In addition, immediate postoperative MRI might show muscle edema due to saline infusion during the arthroscopic surgery, thereby resulting in overestimation of the cross-sectional area at time-zero MRI.

Our study had the following strengths: (1) a relatively large number of patients who underwent MRI at 3 time points: preoperative, at time-zero, and at 6-month follow-up; (2) all MRIs were performed at a single institution using the same protocol and high resolutions; (3) measurements were performed twice by 2 different investigators who were unaware of the outcomes.

However, our study also has several limitations: (1) as timezero MRI was taken the day after surgery, the volume measurement might have increased due to saline edema; (2) although we tried to choose the same scapular plane view as much as possible, small differences during MRI were unavoidable due to patient position and cutting sequence of $3 \mathrm{~mm}$, resulting in

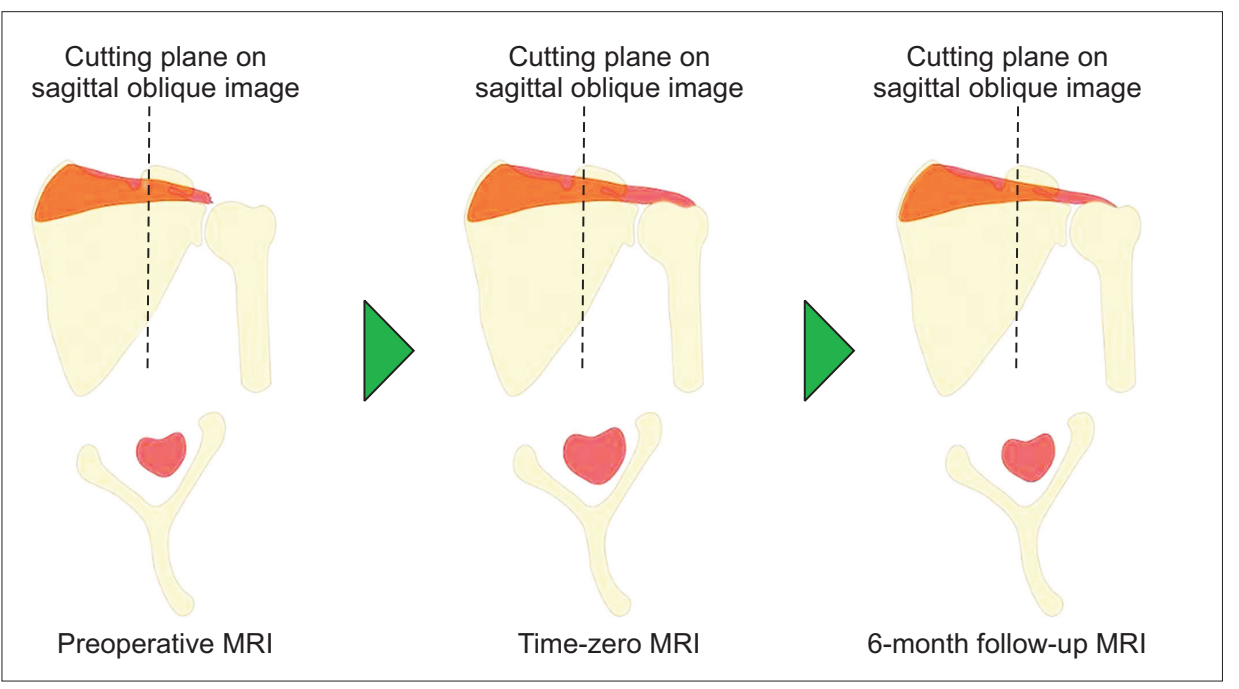

Fig. 5. The schematic drawing presents the cross-sectional area of the supraspinatus muscle on the same scapular plane. The cross-sectional area of the supraspinatus was increased immediately after surgery. However, cross-sectional area was decreased without re-tear 1 month after repair. One of the factors that reduced cross-sectional area is thought to be the medial retraction of repaired tendon. MRI: magnetic resonance imaging. 
slight differences in the 3 cut images from the same patient. In addition, 2 measurements were done by different 2 orthopedic surgeons, leading to the probability of measurement bias; (3) the repair method was not standardized, and a mixture of single row and double rows was used, resulting in the occurrence of some technical bias; (4) the study subjects were limited to those who were willing to undergo MRI at 3 time points; thus, not all patients were included consecutively, thereby introducing sampling bias; (5) we did not compare the same number of patients by tear size, which could lead to a bias in the results; (6) an additional 4 years of follow-up MRI would be more desirable to compare and see the long-term effect of the repair.

\section{Conclusion}

Immediate changes showing morphologic improvement are observed in the Goutallier stage, Thomazeau grade, and crosssectional area of the supraspinatus after successful arthroscopic rotator cuff repair, as measured by MRI from time-zero. Furthermore, the muscle cross-sectional area significantly decreased (11.41\%) from time-zero to 6 months, but the Goutallier stage and Thomazeau grade did not change significantly. The crosssectional area of the initial repair appears to decrease a few months postoperatively, which was possibly due to medial retraction or strained muscle.

\section{References}

1. Goutallier D, Postel JM, Bernageau J, Lavau L, Voisin MC. Fatty infiltration of disrupted rotator cuff muscles. Rev Rhum Engl Ed. 1995;62(6):415-22.

2. Gerber C, Meyer DC, Nuss KM, Farshad M. Anabolic steroids reduce muscle damage caused by rotator cuff tendon release in an experimental study in rabbits. J Bone Joint Surg Am. 2011;93(23):2189-95. doi: 10.2106/JBJS.J.01589.

3. Yoo JC, Ahn JH, Yang JH, Koh KH, Choi SH, Yoon YC. Correlation of arthroscopic repairability of large to massive rotator cuff tears with preoperative magnetic resonance imaging scans. Arthroscopy. 2009;25(6):573-82. doi: 10.1016/j.arthro.2008. 12.015.

4. Liem D, Lichtenberg S, Magosch P, Habermeyer P. Magnetic resonance imaging of arthroscopic supraspinatus tendon repair. J Bone Joint Surg Am. 2007;89(8):1770-6. doi: 10.2106/ JBJS.F.00749.

5. Rubino LJ, Stills HF Jr, Sprott DC, Crosby LA. Fatty infiltration of the torn rotator cuff worsens over time in a rabbit model. Arthroscopy. 2007;23(7):717-22. doi: 10.1016/j.arthro.2007. 01.023.

6. Burkhart SS. Arthroscopic treatment of massive rotator cuff tears. Clin Orthop Relat Res. 2001;(390):107-18.

7. Gerber C, Fuchs B, Hodler J. The results of repair of massive tears of the rotator cuff. J Bone Joint Surg Am. 2000;82(4):50515.

8. Thomazeau H, Boukobza E, Morcet N, Chaperon J, Langlais F. Prediction of rotator cuff repair results by magnetic resonance imaging. Clin Orthop Relat Res. 1997;(344):275-83.

9. Gladstone JN, Bishop JY, Lo IK, Flatow EL. Fatty infiltration and atrophy of the rotator cuff do not improve after rotator cuff repair and correlate with poor functional outcome. Am J Sports Med. 2007;35(5):719-28. doi: 10.1177/0363546506297539.

10. Fuchs B, Gilbart MK, Hodler J, Gerber C. Clinical and structural results of open repair of an isolated one-tendon tear of the rotator cuff. J Bone Joint Surg Am. 2006;88(2):309-16. doi: 10.2106/JBJS.E.00117.

11. Jo $\mathrm{CH}$, Shin JS. Changes in appearance of fatty infiltration and muscle atrophy of rotator cuff muscles on magnetic resonance imaging after rotator cuff repair: establishing new time-zero traits. Arthroscopy. 2013;29(3):449-58. doi: 10.1016/j.arthro. 2012.10.006.

12. Park YB, Ryu HY, Hong JH, Ko YH, Yoo JC. Reversibility of supraspinatus muscle atrophy in tendon-bone healing after arthroscopic rotator cuff repair. Am J Sports Med. 2016;44(4):981-8. doi: 10.1177/0363546515625211.

13. Lee JH, Yoon YC, Jung JY, Yoo JC. Rotator cuff tears noncontrast MRI compared to MR arthrography. Skeletal Radiol. 2015;44(12):1745-54. doi: 10.1007/s00256-015-2228-z.

14. Sugaya H, Maeda K, Matsuki K, Moriishi J. Repair integrity and functional outcome after arthroscopic double-row rotator cuff repair. A prospective outcome study. J Bone Joint Surg Am. 2007;89(5):953-60. doi: 10.2106/JBJS.F.00512.

15. Gerber C, Schneeberger AG, Hoppeler H, Meyer DC. Correlation of atrophy and fatty infiltration on strength and integrity of rotator cuff repairs: a study in thirteen patients. J Shoulder Elbow Surg. 2007;16(6):691-6. doi: 10.1016/j.jse.2007.02.122.

16. Constant CR, Murley AH. A clinical method of functional assessment of the shoulder. Clin Orthop Relat Res. 1987;(214): 160-4.

17. Goutallier D, Postel JM, Gleyze P, Leguilloux P, Van Driessche $\mathrm{S}$. Influence of cuff muscle fatty degeneration on anatomic and functional outcomes after simple suture of full-thickness tears. J Shoulder Elbow Surg. 2003;12(6):550-4. doi: 10.1016/ S1058274603002118.

18. Matsumoto F, Uhthoff HK, Trudel G, Loehr JF. Delayed tendon reattachment does not reverse atrophy and fat accumulation of the supraspinatus--an experimental study in rabbits. J Orthop Res. 2002;20(2):357-63. doi: 10.1016/S0736-0266(01)000936.

19. Shimizu T, Itoi E, Minagawa H, Pradhan RL, Wakabayashi I, Sato K. Atrophy of the rotator cuff muscles and site of cuff tears. Acta Orthop Scand. 2002;73(1):40-3. doi: 10.1080/ 000164702317281387.

20. Goutallier D, Postel JM, Bernageau J, Lavau L, Voisin MC. Fatty 
muscle degeneration in cuff ruptures. Pre- and postoperative evaluation by CT scan. Clin Orthop Relat Res. 1994;(304):7883.

21. McCarron JA, Derwin KA, Bey MJ, et al. Failure with continuity in rotator cuff repair "healing". Am J Sports Med.
2013;41(1):134-41. doi: 10.1177/0363546512459477.

22. Baring TK, Cashman PP, Reilly P, Emery RJ, Amis AA. Rotator cuff repair failure in vivo: a radiostereometric measurement study. J Shoulder Elbow Surg. 2011;20(8):1194-9. doi: 10.1016/j.jse.2011.04.010. 